\title{
Über die perkutane Resorption einiger Ester der Salizylsäure.
}

Von

E. Impens, Elberfeld.

(Mit 2 Textfiguren.)

Die bei gewöhnlicher Temperatur flüssigen Ester der Salizylsäure, selbst diejenigen chemiseh nabestehender homologer Alkohole, weisen auffallende Unterschiede in der Ausgiebigkeit ihrer Resorption durch die intakte Haut auf.

Die einfache Prüfung des Harns mit Eisenchlorid genügt, um diese Tatsache zu veranschaulichen. Während schon kurze Zeit nach Einreibung von Methylsalizylat z. B. eine intensive violette Färbung die Anwesenheit nicht unbeträchtlicher Mengen Salizylsäure in dem Harn verrät, kommt diese Reaktion nach Applikation von Amylsalizylat nur langsam und zaghaft zum Vorschein.

Es unterliegt keinem Zweifel, dass solche Differenzen in der perkutanen Aufnahmefähigkeit die Brauchbarkeit dieser Verbindungen, welche eine ausgedehnte Verwendung als externe Antirheumatica finden, stark beeinflussen müssen, denn ihre therapeutische Wirksamkeit rührt lediglich von der resorbierten Salizylsäure her, und wohl kaum, wie einige Kliniker es vermutet haben, von einer gelegentlichen Reizwirkung oder von der bei der Einreibung verübten Massage.

Der Zweck der vorliegenden Untersuchungen war nun: erstens, möglichst quantitativ zu bestimmen, wieviel von den eingeriebenen Estern durch die Tegumente in die Körpersäfte übergeht; zweitens, zu prüfen, welche von den chemisch-physikalischen oder physiologischen Eigenschaften der betreffenden Präparate als begünstigende Faktoren für die perkutane Resorption anzusehen sind.

Eines der wirksamsten Schutzmittel der Haut gegen das Eindringen sowie gegen die schädliche Einwirkung mit ihr in Kontakt gebrachter fremder Stoffe besteht in ibrem Reichtum an Fett. Die 
Sebumdrüsen erfüllen mit ihrem Sekret nicht allein den Raum zwischen dem Haarschaft und dem Hals der Haarfollikel, sondern dieser Talg verbreitet sich auch über die ganze Fläche der Epidermis und durchtränkt die Zellager des Stratum corneum. Jede Substanz, welche auf die Haut eingerieben wird, muss, um die äussere Schicht der Epidermis zu benetzen, und um in die Drüsen- und Haarfollikelöffnungen zu gelangen, sich in diesem Talg oder Sebum auflösen können. Ist diese erste Barrière überwunden, so findet sich bald eine zweite in dem Stratum granulosum, dessen mit Eleidintropfen gefüllte Zellen einen Schutzwall für das Stratum malpighii bilden, und schliesslich eine letzte in den Fettzelleneinlagen der tieferen Cutisschichten, welche mit den Fettlagern des Unterbautbindegewebes den an gewissen Körperstellen so mächtig entwickelten Panniculus adiposus ausmachen.

Die Medikamente, welche von der Haut aufgenommen werden sollen, müssen demnach vor allem fett- oder öllöslich sein. Für die Resorption günstig ist aber ebenfalls eine gewisse Wasserlöslichkeit, damit die Substanzen von dem Hauttalg oder -fett nicht zu lange festgehalten werden; denn sollen sie zu der Blutbahn ibren weiteren Weg finden oder zu Organen gelangen, welche in dem Plasma der Lymphspalten eingebettet sind, wie die Nervenendigungen in der Cutis, oder welche noch tiefer liegen, wie z. B. die peripheren Nervenstränge und die unter der Haut liegenden Muskeln, wo sie ihre Wirkung zu entfalten haben, so darf ihr Übergang aus dem Fett in die Lymphe nicht zu sehr erschwert werden. Da spielt der Teilungskoeffizient von Öl zu Wasser eine grosse Rolle.

Bei den hier in Betracht kommenden Estern kän dieser Übergang durch eine mehr oder weniger leichte Alkali- oder Wasserspaltbarkeit unterstützt werden, da die resultierende Salizylsäure und noch viel mehr ihr Natronsalz leicht vom Plasma dem Hautfett entzogen werden.

Weiter sind als Hilfsfaltoren für die perkutane Resorption noch die innere Reibung oder Viskosität und die Kapillarität ins Auge zu fassen, weil man mit Recht annehmen darf, dass die Leichtigkeit, mit welcher die eingeriebenen Substanzen sich in den Poren der Haut bewegen, und die Geschwindigkeit, mit welcher die Tegumente imbibiert werden, von Einfluss auf die Höhe der resorbierten Mengen sein könnten. Endlich bleibt noch zu bemerken, dass in den Drüsengängen sich Luft befindet, welche das Weiterdringen der Medikamente 
erschwert; dieses Hindernis wird von den flüchtigen Substanzen, wie Chloroform, Methyljodid, am leichtesten überwunden. Sie sättigen die Lufträume mit ihrem Dampf; dieser wird dann an den Wandungen entweder in dem Talg wieder aufgelöst oder von den Gewebssäften aufgenommen. Es kommt demnach für die perkutane Resorption auch die Dampfspannung unter Umständen in Betracht.

In den Kreis dieser Untersuchungen wurden drei in die Therapie schon längere Zeit eingeführte Salizylderivate, nämlich das Gaultheriaöl (Methylsalizylat), das Ulmaren (Amylsalizylat), das Mesotan (Methoxymethylsalizylat), und ausserdem ein neues Präparat, welches klinisch noch nicht geprüft war, der primäre Salizylsäureester des Äthylenglykols gezogen. Letztere Substanz hat folgende Konstitution: $\mathrm{CH}_{2}-\mathrm{OH}$

$\mathrm{CH}_{2} \mathrm{O} \cdot \mathrm{COC}_{6} \mathrm{H}_{4} \mathrm{OH}$

und wird aus Glykol und Salizylsäure oder aus Natriumsalizylat und Äthylenchlorhydrin dargestellt. Eine verwandte Verbindung, das Glykoldisalizylat $\mathrm{CH}_{2}-\mathrm{O} \cdot \mathrm{COC}_{6} \mathrm{H}_{4} \mathrm{OH}$ $\mathrm{CH}_{2}-\mathrm{O} \cdot \mathrm{COC} \mathrm{C}_{6} \mathrm{H}_{4} \mathrm{OH}$ ist von Bondzinsky im Jahre 1897 auf sein Verhalten im Organismus untersucht worden (Arch. f. exp. Path. und Pharm. Bd. 38 S. 88). Nach den Angaben dieses Verfassers wird dieses Präparat etwas über $40 \%$ vom Darm aus resorbiert, während ungefähr $19 \%$ in dem Kot zurückgefunden werden. Eine Verwendung als Ersatz für das Natriumsalizylat im internen Gebrauch hat das Glykoldisalizylat nicht gefunden.

Da der primäre Salizylsäureester des Glykols, im Gegensatz zu dem Disalizylat, bei gewöhnlicher Temperatur ein öl bildet, kommt er für die interne Therapie gar nicht in Betracht; er eignet sich aber, besonders durch seine Geruch- und Reizlosigkeit, für die äusserliche Anwendungsart und hat wegen seiner diesbezüglichen guten Eigensehaften eigentlich den Anlass zu dieser kleinen Arbeit gegeben.

Der Gang der Untersuchungen wurde derart angeordnet, dass die genannten Substanzen zuerst auf ihre Wasser- und Öllöslichkeit, jhre Spaltbarkeit durch Wasser und schwache Alkalien, ihre Viskosität und Oberflächenspannung, dann auf ihre Reizwirkung der Haut gegenüber geprüft wurden. Diese letzte physiologische Eigenschaft spielt bei der perkutanen Resorption durch die lokale Gefässerweiterung und Kreislaufanregung, welche sie hervorruft, eine nicht $\mathrm{zu}$ vernachlässigende Rolle, wie wir in der Folge sehen werden. 
Endlich wurde die Grösse der resorbierten Estermenge mit Hilfe der Salizylsäurebestimmung im Harn geschätzt.

1. Löslichkeit in Olivenöl: Die Methyl-, Amyl- und Metboxymethylsalizylate mischen sich mit Olivenöl in jedem Verhältnis. Das Glykolmonosalizylat ist in diesem Vehikel weniger löslich; immerhin beträgt die Löslichkeit bei $22^{\circ}$ noch immer ca. $16 \%$.

2. Löslichkeit in Wasser: Die Wasserlöslichkeit, welche im Gegensatz zu der Öllöslichkeit ziemlich spärlich ist, konnte nur auf analytischem Wege festgestellt werden. $200 \mathrm{ccm}$ der möglichst schnell angefertigten und klar filtrierten gesättigten Esterlösungen wurden mit einer genügenden Menge Barythydrat versetzt und sechs Stunden am Rückflusskübler gekocht, um den gelösten Ester zu verseifen. Nach dem Erkalten wurde mit Salzsäure angesäuert und bis auf $1000 \mathrm{ccm}$ mit Wasser genau verdünnt. In $100 \mathrm{cem}$ dieser Lösung wurde nun die Salizylsäure nach der Fr e y er'schen Methode bestimmt (Zeitschr. f. an. Chem. Bd. 38 S. 292).

Diese Methode beruht auf der Tatsache, dass Salizylsäure, indem sie sich in Tribromphenol umwandelt, drei Bromatome zu fixieren vermag, wenn man freies Brom in wässeriger Lösung auf sie einwirken ässt. Da sich zu gleicher Zeit drei Bromwasserstoffsäuremolekülen bilden, entspricht jede Moleküle Salizylsäure einem Verbrauch von sechs Atomen Brom.

Zur Ausführung der Analyse setzt man zu der in Frage kommenden Flüssigkeit eine gegebene Menge einer bromsauren KaliBromkalilösung, deren Bromtiter mit 1/10 norm. Thiosulfat genau festgestellt ist, zu, säuert mit Salzsäure kräftig an, lässt 5 Minuten lang einwirken, fügt eine hinreichende Menge einer $10 \%$ igen Jodkaliumlösung binzu und titriert das freigewordene Jod mit Hilfe einer ${ }^{1_{10}}$ norm. Thiosulfatlösung zurück. Die Differenz zwischen der abgelesenen Kubikzentimeterzahl und derjenigen, welche der zugesetzten bromsauren Kali-Bromkalilösung entspricht, ergibt die gesuchte Salizylsäuremenge: jeder Kubikzentimeter der 1/10 norm. Thiosulfatlösung repräsentiert $0,00239 \mathrm{~g}$ Salizylsäure.

Anstatt den Ester zu verseifen, kann man ihn auch direkt als solchen durch Bromierung bestimmen; da aber die Ester nur zwei Atome Brom fixieren, haben sie nur einen Verbrauch von vier Bromatomen, und jeder Kubikzentimeter 1/xo norm. Thiosulfatlösung entspricht $0,00345 \mathrm{~g}$ Salizylsäure. 
Die Wasserlöslichkeit wurde auf beide Weisen (ausser für Mesotan) festgestellt, und die gefundenen Werte waren gut übereinstimmend.

Nach meinen Versuchen beträgt nun die Wasserlöslichkeit bei 22 Grad:

$$
\begin{aligned}
& \text { des Methydsalizylats . . . . 0,0725\% } \\
& \text { des Amylsalizylats . . . . . . 0,0039\% } \\
& \text { des Mesotans. . . . . 0,0913\% } \\
& \text { des Glykolmonos }
\end{aligned}
$$

Für das Mesotan wurde die Bestimmung nur durch Verseifung gemacht, weil dieser Ester zu labil ist; die Zersetzlichkeit diesei Substanz in Gegenwart von Wasser ist so gross, dass während des Auflösens schon ein Teil sich spaltet; die durch Wasser gespaltene Menge, welche man durch Titration der entstandenen Salizylsäure bestimmen kann, muss dann abgezogen werden, um die richtige Löslichkeitszahl zu bekommen.

Das Amylsalizylat ist am wenigsten löslich in Wasser; stellen wir seine Löslichkeit gleich 1 , so ist diejenige des Methylsalizylats gleich 18,5, diejenige des Mesotans ca. 23 und diejenige des Glykolmonosalizylats 248 .

Das Glykolmonosalizylat, welches von den vier Estern in Öl am wenigsten löslich ist, ist in Wasser bei weitem das löslichste; es besitzt demnach den günstigsten Teilungskoeffizienten von Öl zu Wasser.

Nehmen wir die Löslichkeit der drei anderen Ester in Olivenöl als $100 \%$ betragend an, so haben wir folgende Teilungskoeffiziente:

Glykolmonosalizylat . . . . . . ca. 16,5

Mesotan . . . . . . . . . 1095

Methylsalizylat . . . . . . 1380

Amylsalizylat . . . . . . . 25600

Berücksichtigt man nur diese Werte, so wird man finden, dass das Glykolmonosalizylat 67 mal leichter als das Mesotan, das Mesotan 1,2 mal leichter als das Gaultherialöl, letzteres endlich 18 mal leichter als das Ulmaren aus dem Öl in Wasser übergeht.

Ich brauche nicht auf die Bedeutung einzugehen, welche so beträchtliche Unterschiede für den Durchgang der betreffenden Substanzen durch die Haut haben.

3. Spaltbarkeit durch Wasser. Die Salizylate des Glykols, der Metbyl- and Amylalkohole sind Wasser gegenüber sehr 
beständig; anders liegt der Fall für das Mesotan. Dieser Ester wird durch das Wasser prompt in seine Komponenten, Salizylsäure, Formaldehyd und Methylalkohol, gespalten. Der Gang dieser Zersetzung lässt sich bequem verfolgen durch einfache Titration der entstandenen Säuremengen. $2 \mathrm{~g}$ Mesotan wurden um $12^{\mathrm{b}} 30^{\prime}$ mit $500 \mathrm{ccm}$ Wasser bei $23^{\circ}$ gemischt und dauernd geschüttelt. Nach bestimmten Zeitintervallen wurden je $50 \mathrm{ccm}$ entnommen und mit $1 / 5$ norm. Natronlauge titriert: Die zur Neutralisation nötige Kubikzentimeterzahl der Lauge betrug: um $1^{\text {h }} 30^{\prime} 0,3 \mathrm{ccm} ;$ um $2^{\mathrm{h}} 30^{r}$ $0,6 \mathrm{ccm} ;$ um $3^{\mathrm{h}} 30^{\prime} 0,9 \mathrm{ccm}$. Am folgenden Morgen um $8^{\mathrm{h}} 30^{\prime}$ $3,2 \mathrm{ccm}$.

Von den $2 \mathrm{~g}$ Mesotan waren demnach zersetzt: nach 1 Stunde $0,1092 \mathrm{~g}$ oder $5,46 \%$; nach 2 Stunden $0,218 \mathrm{~g}$ oder $10,9 \%$; nach 3 Stunden $0,327 \mathrm{~g}$ oder $16,3 \%$, endlich nach 20 Stunden $1,164 \mathrm{~g}$ oder $58,2 \%$.

4. Verseifung durch Alkalien. Während das Mesotan eine Ausnahmestellung in betreff der Wasserhydrolyse einnimmt, finden wir bei der Spaltung durch schwache Alkalien, durch Darmsoda ( $1 \%$ Krist. - Soda) lediglich Unterschiede quantitativer Natur. Alle vier Ester wurden von der Darmsoda angegriffen; die Geschwindigkeit der Verseifung variiert aber in weiten Grenzen.

Um die Verseifungsgeschwindigkeit zu schätzen, wurde eine bestimmte Menge des Esters mit 500 cem Darmsoda, deren Alkaligehalt mit $1 / 10$ norm. Schwefelsäure und Methylorange festgestellt war, geschüttelt und die Alkaleszenzabnahme stündlich durch Titration kontrolliert.

Die Ergebnisse dieser Versuche sind in folgender Zusammenstellung niedergelegt:

a) Methylsalizylat. 2,5 g Ester werden mit $500 \mathrm{ccm}$ Darmsoda bei $17^{\circ}$ C. geschüttelt; diese Menge Alkali entspricht $359 \mathrm{ccm}$ $1 / 10$ norm. $\mathrm{SO}_{4} \mathrm{H}_{2}$ und würde theoretisch genügen, um $5,46 \mathrm{~g}$ Ester zu verseifen.

\begin{tabular}{|c|c|c|c|}
\hline & $\begin{array}{l}\text { Titer von } 25 \mathrm{ccm} \text { in } \\
1 / 10 \text { norm. } \mathrm{SO}_{4} \mathrm{H}_{2}\end{array}$ & $\begin{array}{c}\text { Totale verseifte } \\
\text { Menge }\end{array}$ & $\begin{array}{l}\text { Proz. verseifte } \\
\text { Menge }\end{array}$ \\
\hline$r$ der Mischung . & $17,95 \mathrm{ccm}$ & - & - \\
\hline ch 1 Stunde. . & $17,55 \mathrm{ccm}$ & $0,121 \mathrm{~g}$ & $2,2 \%$ \\
\hline ch 2 Stunden & $17,25 \mathrm{ccm}$ & $0,213 \mathrm{~g}$ & $3,9 \%$ \\
\hline ach 3 Stunden & $17,00 \mathrm{ccm}$ & $0,289 \mathrm{~g}$ & $5,2 \%$ \\
\hline ch 4 Stunden & $16,75 \mathrm{ccm}$ & $0,365 \mathrm{~g}$ & $6,6 \%$ \\
\hline ch 5 Stunden. & $16,50 \mathrm{ccm}$ & $0,441 \mathrm{~g}$ & $8,0 \%$ \\
\hline ch 6 Stunden. & $16,25 \mathrm{ccm}$ & $0,516 \mathrm{~g}$ & $9,4 \%$ \\
\hline
\end{tabular}


Der Prozentsatz wird auf die durch $500 \mathrm{ccm}$ Darmsoda verseifbare Menge Methylsalizylat berechnet, also 5,46 g; und nicht auf die in Wirklichkeit zugesetzte Menge von $2,5 \mathrm{~g}$.

b) Amylsalizylat. $3,42 \mathrm{~g}$ Ester werden mit $500 \mathrm{ccm}$ derselben Darmsoda wie in a) geschüttelt. Diese Menge hätte 7,48 $\mathrm{g}$ Amylsalizylat verseifen können.

$\begin{array}{lccc} & \begin{array}{c}\text { Titer von } 25 \mathrm{ccm} \text { in } \\ 1 / 10 \text { norm. } \mathrm{SO}_{4} \mathrm{H}_{2}\end{array} & \begin{array}{c}\text { Totale verseifte } \\ \text { Menge }\end{array} & \begin{array}{c}\text { Proz. verseifte } \\ \text { Menge }\end{array} \\ \text { Vor der Mischung . . . } & 17,95 \mathrm{ccm} & - & - \\ \text { Nach 1 Stunde. . . . . } & 17,90 \mathrm{ccm} & 0,0208 \mathrm{~g} & 0,27 \mathrm{o} / 0 \\ \text { Nach 2 Stunden . . . . } & 17,85 \mathrm{ccm} & 0,0416 \mathrm{~g} & 0,55 \% \\ \text { Nach 3 Stunden . . . . } & 17,80 \mathrm{ccm} & 0,0624 \mathrm{~g} & 0,83 \% \\ \text { Nach 4 Stunden . . . . } & 17,75 \mathrm{ccm} & 0,0832 \mathrm{~g} & 1,1 \% \\ \text { Nach 5 Stunden . . . . } & 17,65 \mathrm{ccm} & 0,1248 \mathrm{~g} & 1,6 \% \\ \text { Nach 6 Stunden . . . . } & 17,55 \mathrm{ccm} & 0,1664 \mathrm{~g} & 2,21 \%\end{array}$

c) Mesotan. $3 \mathrm{~g}$ des Esters werden mit $500 \mathrm{cem}$ Darmsoda geschüttelt; den $500 \mathrm{ccm}$ Darmsoda entsprechen $6,533 \mathrm{~g}$ Mesotan.

$\begin{array}{lccc} & \begin{array}{c}\text { Titer von } 25 \mathrm{ccm} \text { in } \\ 1 / 10 \text { norm. } \mathrm{SO}_{4} \mathrm{H}_{2}\end{array} & \begin{array}{c}\text { Totale verseifte } \\ \text { Menge }\end{array} & \begin{array}{c}\text { Proz. verseift } \\ \text { Menge }\end{array} \\ \text { Vor der Mischung . . . } & 17,95 \mathrm{ccm} & - & - \\ \text { Nach 1 Stunde. . . . . } & 17,10 \mathrm{ccm} & 0,3094 \mathrm{~g} & 4,7 \% \\ \text { Nach 2 Stunden . . . . } & 16,05 \mathrm{~cm} & 0,692 \mathrm{~g} & 10,6 \% \\ \text { Nach 3 Stunden . . . . } & 14,85 \mathrm{ccm} & 1,129 \mathrm{~g} & 17,3 \% \\ \text { Nach 4 Stunden . . . . } & 13,65 \mathrm{ccm} & 1,564 \mathrm{~g} & 24,0 \% \\ \text { Nach 5 Stunden . . . . } & 12,55 \mathrm{ccm} & 1,965 \mathrm{~g} & 30,1 \% \\ \text { Nach 6 Stunden . . . . } & 11,7 \mathrm{ccm} & 2,27 \mathrm{~g} & 34,8 \%\end{array}$

d) Glykolmonosalizylat. Es werden $3 \mathrm{~g}$ des Esters mit $500 \mathrm{cem}$ Darmsoda bei $17^{\circ} \mathrm{C}$. geschüttelt; durch diese Menge Darmsoda hätten $6,533 \mathrm{~g}$ verseift werden können.

$\begin{array}{lccc} & \begin{array}{c}\text { Titer von } 25 \mathrm{~cm} \text { in } \\ 1 / 10 \text { norm. } \mathrm{SO}_{4} \mathrm{H}_{2}\end{array} & \begin{array}{c}\text { Totale verseifte } \\ \text { Menge }\end{array} & \begin{array}{c}\text { Proz. verseifte } \\ \text { Menge }\end{array} \\ \text { Vor der Mischung . . . } & 17,95 \mathrm{ccm} & - & - \\ \text { Nach 1 Stunde. . . . . } & 17,10 \mathrm{ccm} & 0,309 \mathrm{~g} & 4,7 \% \\ \text { Nach 2 Stunden . . . . } & 16,45 \mathrm{ccm} & 0,546 \mathrm{~g} & 8,3 \% \\ \text { Nach 3 Stunden . . . . } & 15,85 \mathrm{ccm} & 0,764 \mathrm{~g} & 11,7 \% \\ \text { Nach 4 Stunden . . . . } & 15,30 \mathrm{ccm} & 0,965 \mathrm{~g} & 14,7 \% \\ \text { Nach 5 Stunden . . . . } & 14,95 \mathrm{ccm} & 1,092 \mathrm{~g} & 16,7 \% \\ \text { Nach 6 Stunden . . . . } & 14,60 \mathrm{ccm} & 1,22 \mathrm{~g} & 18,6 \%\end{array}$

Die Unterschiede in der Verseifungsgesehwindigkeit durch schwache Alkalien lassen sich aus folgendem Diagramm deutlicher erkennen: 


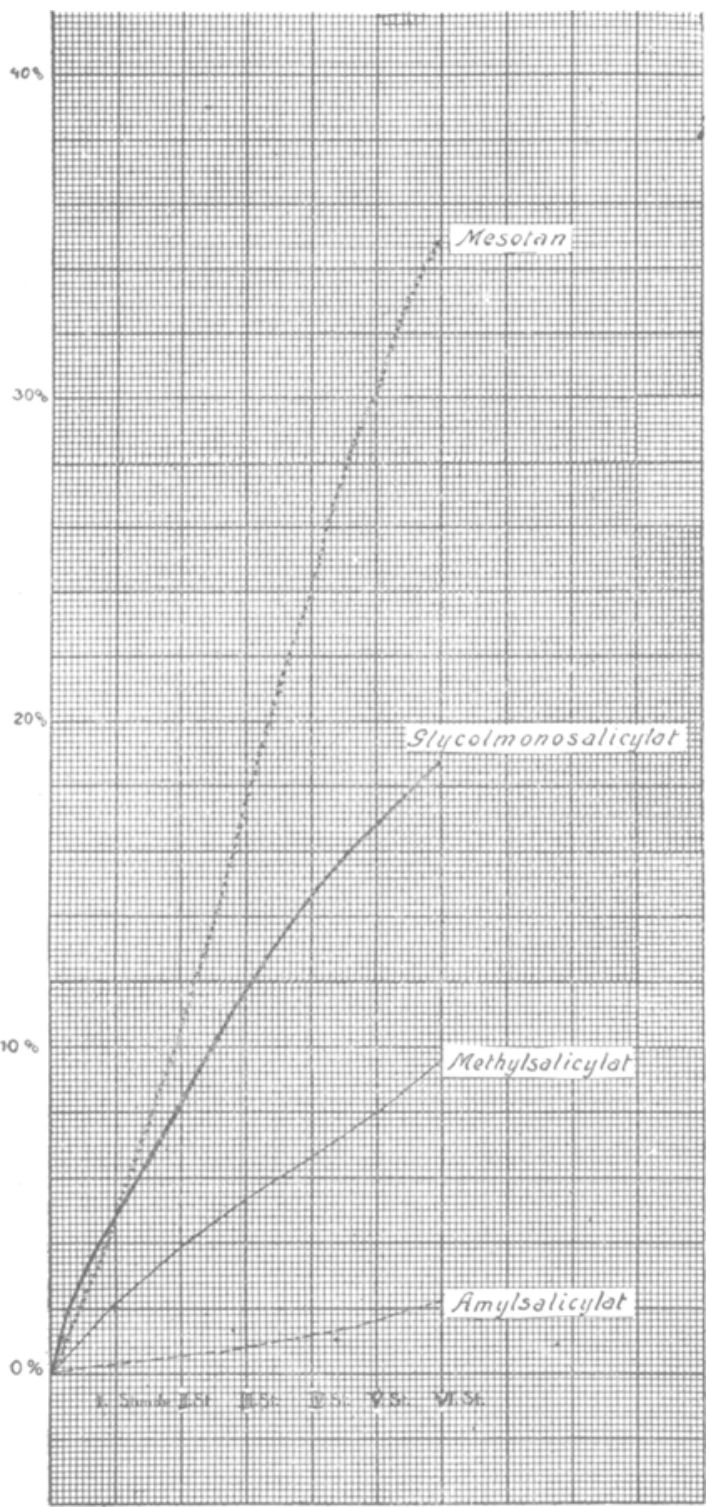

Fig. 1.

Die beträchtlichen Unterschiede, welche diese Salizylsäureester in ibrem Verhalten zu. den Alkalien aufweisen, rühren nur teilweise von der Natur der alkoholischen Komponente her; die Hauptursache ist wohl in der Wasserlöslichkeit $\mathrm{zu}$ suchen, indem die Begegnung der Molekülen mit denjenigen des Alkalis bei den wasserlöslichen Estern unvergleichlich viel bäufiger stattfindet. Das in Wasser fast unlösliche Ulmaren wird von der Darmsoda kaum angegriffen; das Gaultheriaöl, welches in Wasser ca. $20 \mathrm{mal} \mathrm{lös-}$ licher ist, wird in der ersten Einwirkungsstunde, welche für uns allein in Betracht kommt, etwa $8 \mathrm{mal}$ rascher zersetzt; das Glykolmonosalizylat, welches in Wasser noch viel löslicher ist, zerfällt $17 \mathrm{mal}$ schneller als das Ulmaren. Das Mesotan aber macht eine Ausnahme: obgleich es nicht viel löslicher als das Methylsalizylat ist, verseift es anfangs ebenso rasch als das Glykolmonosalizylat, später sogar noch in stärkerem Maasse. Der Grund dieses abweichenden Verhaltens liegt in der so leichten Zersetzlichkeit des Mesotans durch Wasser. 
In der Tat sehen wir, dass das Wasser nach einer Stunde $5,46 \%$ des zugefügten Mesotans verseift, während Darmsoda in der gleichen Zeit 15,4\% derselben Menge Ester spalten würde, also nicht ganz das Dreifache.

Es ist nicht von der Hand zu weisen, dass die Verbindungen, welche am leichtesten verseifen, am schnellsten aus einer Fett- oder Öllösung in ein schwach alkalisches Medium, wie die Lymphe z. B., übergehen müssen. Die Alkalispaltbarkeit kann in dieser Hinsicht der Wasserlöslichkeit zu Hilfe kommen und erleichtert manchen Estern, welche in Wasser sehr schlecht löslich sind, den Übergang vom Öl in wässerige Flüssigkeiten.

5. Innere Reibung und Kapillarität. Ausser diesen bis jetzt untersuchten Eigenschaften mehr chemischer Natur gibt es noch rein physikalische, welche die Resorption der Medikamente durch die Haut fördern oder verzögern können. Es ist bekannt, dass das Glyzerin, das Olivenöl die perkutane Resorption der in ihnen gelösten oder suspendierten Substanzen stark verlangsamen. Diese Tatsache ist nicht allein auf die Verdünnung zurückzuführen, sondern auf die starke Viskosität der benannten Flüssigkeiten. In diesen dickflüssigen Medien werden die Bewegungen der Flüssigkeitsehichten sehr gehemmt und dadurch die Zahl der Molekülen, welche durch ibre intime Berührung mit den Hautelementen zur Resorption gelangen können, reduziert. Anderseits darf man annehmen, dass ein rasches Eindringen der Substanzen zwischen die Unebenheiten der Epidermisoberfläche und in die Öffnungen verschiedener Art, welche das Stratum corneum aufweist, die Resorption beschleunigen und erleichtern muss.

Aus diesem Grunde habe ich die Ester, welche uns hier beschäftigen, auf ihre innere Reibung und auf ihre Kapillarität gepriuft.

Die Viskosität wurde mit Hilfe des Ostwaldschen Viskosimeters gemessen.

Die Ergebnisse sind folgende:

Ausflusszeit bei $17^{\circ}$ : für Gautheriaöl . . . 5 Min. 2 Sek.

für Amylsalizylat . . 10 Min. 40 Sek.

für Mesotan . . . 11 Min. 18 Sek.

für Glykolmonosalizylat. 4 Stri. 30 Min.

Wie man sieht, zeigen die Ester auch hier grosse Unterschiede; das Glykolmonosalizylat ist sehr dickflüssig, und es wirkt zweifellos 
diese Eigenschaft den anderen für die Resorption dieser Substanz so günstigen Verhältnissen etwas entgegen. Die geringe Viskosität des Methylsalizylats kann dagegen die Aufnahme dieses Esters durch die Haut unterstützen.

Die Kapillaritätsversuche wurden mit Glasröhrehen, welche ein Lumen von $0,42 \mathrm{~mm}$ hatten, angestellt, ùnd zwar derart, dass man die Höhe, welche die Ester in der Kapillare erreichen konnten, wenn man dieselbe senkrecht in die Flüssigkeit tauchte, maass.

Diese Höhen sind bei $17^{\circ}$ : für Gaultheriaöl . . . $4,2 \mathrm{~cm}$ für Mesotan . . . . $2,3 \mathrm{~cm}$ für Glykolmonosalizylat $3,9 \mathrm{~cm}$

Für das Ulmaren wurde ein Röhrchen von $0,36 \mathrm{~mm}$ Lichtweite gebraucht. Die Höhe erreichte darin $3,3 \mathrm{~cm}$.

Diese Versuche fallen für das Gaultheriaöl wieder am günstigsten aus. Diese Substanz hat die Fähigkeit, sich in die Gewebsspalten einzusaugen, also die Epidermis prompt zu benetzen, am ausgeprägtesten; diese Eigenschaft, wenn auch im geringerem Grade, kommt auch dem Glykolester zu und hebt zum Teil die Nachteile seiner starken Viskosität auf.

6. Flüchtigkeit. Dieselbe wurde nach der dynamischen Methode, wie sie in Ostwald-Luther's physiko-chemischen Messungen S. 270 (II. Aufl.) beschrieben ist, gemessen, und zwar bei einer Temperatur von annähernd $37^{\circ}$.

Auf diese Weise wurde gefunden, dass 10 Liter Luft

$$
\begin{array}{ll}
0,0205 & \mathrm{~g} \text { Gaultheriaöl } \\
0,0110 & \mathrm{~g} \text { Amylsalizylat } \\
0,0069 & \mathrm{~g} \text {. Mesotan } \\
0,00066 & \mathrm{~g} \text { Glykolmonosalizylat }
\end{array}
$$

als Dampf fortführen.

Diese Werte sind sehr gering, und der Einfluss der Flüchtigkeit auf die perkutane Resorption dieser Ester ist, ausser für das Gaultheriaöl, praktisch zu vernachlässigen.

7. Reizwirkung. Das Mesotan ist, wie wir es gesehen haben, Wasser gegenüber sehr empfindlich; bleibt eine Probe dieser Substanz längere Zeit der Einwirkung der Feuchtigkeit ausgesetzt, so tritt allmählich eine Zersetzung ein, welche dadurch wahrnehmbar wird, dass der Ester einen deutlichen Formaldehydgeruch verbreitet und ausserdem die Eigenschaft angenommen hat, auf der Haut mehr 
oder weniger ausgeprägte Reizerscheinungen hervorzurufen, während das unzersetzte Mesotan nur einen sehr geringen, schwach an Gaultheriaöl erinnernden Geruch besitzt und auf der Haut keine nennenswerte Reizwirkung ausübt.

Das zersetzte Mesotan erzeugt am Kaninchenohr eine entzündliche Schwellung, wie Dreser es gezeigt hat (Ther. Monatshefte 1903). Gaultheriaöl, Ulmaren, unzersetztes Mesotan und Glykolmonosalizylat haben diese Wirkung nicht. Die drei ersteren Verbindungen können unter Umständen eine gewisse Hyperämie hervorrufen, die letzte dagegen scheint für die Haut des Kaninchenohres ganz indifferent zu sein. Auch beim Menschen habe ich nie Reizerscheinungen nach Einreibung von Glykolmonosalizylat gesehen.

Ein deutlicher Einfluss der Reizwirkung auf die perkutane Resorption kann sich demnach nur für das zersetzte Mesotan geltend machen.

8. Resorption. Zur Schätzung der perkutanen Resorption wurde die in dem Harn nach Einreibung einer gewogenen Menge des Esters ausgeschiedene Salizylsäure bzw. Salizylursäure so genau wie nur möglich bestimmt.

Die Versuche wurden am gesunden Menschen gemacht und an derselben Person; als Einreibungsstelle wählte ich die vordere Fläche des Rumpfes. Die Salizylsäurebestimmung nahm ich auf folgende Weise vor: Der gesammelte Harn wurde mit Kochsalz gesättigt, mit Schwefelsäure angesäuert und mit Äther extrahiert. Der Äther wurde mit einer Sodalösung ausgezogen, die Sodalösung wieder angesäuert, mit Talkum versetzt und filtriert. Das klare Filtrat oder, im Falle eines stark salizylsäurehaltigen Harns, ein aliquoter Teil desselben wurde dann, nach Sättigung mit NaCl, mit je $100 \mathrm{ccm}$ eines Gemisches von gleichen Teilen Äther mit Petroläther so lange ausgeschüttelt, bis in einer Probe der Äthermischung nach dem Verdunsten bei Zimmertemperatur und Aufnahme des Rückstandes mit einem Tropfen Wasser mit stark verdünnter Eisenchloridlösung keine Salizyl- oder Salizylursäure mehr nachweisbar war. Die gesammelten Äther-Petroläthermengen wurden danach mit einer wässerigen Sodalösung 3 mal behandelt, und in dem vom Äther befreiten, mit Salzsäure neutralisierten wässerigen Auszug wurde schliesslich die Salizylsäure bzw. Salizylursäure nach Freyer bestimmt. 
Die auf diese Weise erhaltenen Werte sind nicht direkt brauchbar, weil der normale Harn ebenfalls Bestandteile enthält, welche Brom zu binden vermögen. Aus diesem Grunde wurde in dem 24 stündigen Harn eines oder zweier Tage vor der Einreibung nach identischer Behandlang die Brombindung bestimmt und die gewonnenen Werte von den obigen abgezogen.

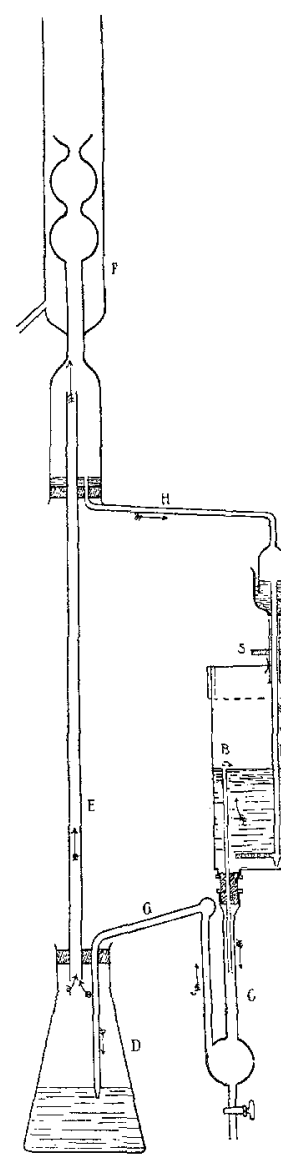

Fig. 2.

Dieses normale Brombindungsvermögen des Harns wechselt nach der Nahrung; es empfiehlt sich demnach, wäbrend der Versuche eine möglichst gleichartig zusammengesetzte Kost nehmen zu lassen. Bei Berücksichtigung dieses Faktors, schwanken die Normalwerte nur in geringen Grenzen.

Abgesehen von dieser Korrektur, ist bei der Berechnung der Salizylsäuremenge aus dem Verbrauch an $1 / 10$ norm. Thiosulfatlösung eine zweite Korrektur anzubringen. In der nach obigem Extraktionsverfahren erhaltenen wässerigen Lösung ist nämlich Salizylsäure neben Salizylursäure enthalten. Während nun Salizylsäure drei Bromatome bindet, um sich in Tribromphenol umzuwandeln, verbraucht Salizylursäure nur zwei, weil es in Dibromsalizylursäure übergeht. Da nach Dreser die Salizylsäure annähernd $\mathrm{zu} 50 \%$ als Salizylursäure in dem Harn erscheint, werden wir mit genügender Genauigkeit (es handelt sich ja doch nur um Vergleichszahlen, nicht um absolute Werte) die Menge der Salizylsäure, der gepaarten sowobl wie der ungepaarten, bekommen, wenn wir die Kubikzentimeterzahl der verbrauchten Thiosulfatlösung mit $0,00276^{\circ}$ multiplizieren (anstatt 6 Atome Brom rechnen wir auf eine Moleküle Salizylsäure 5). In einer späteren Publikation werde ich zeigen, wie man mit Hilfe von Chloroform die beiden Säuren im Harn trennen kann und imstande ist, sie separat zu bestimmen.

Bevor ich jetzt die Ergebnisse der Resorptionsversuche mitteile, 
möchte ich noch erwähnen, dass die Extraktion des Harns mit Äther nicht durch Ausschütteln, sondern in einem zu dem Zweck von mir konstruierten Extraktionsapparat vorgenommen wurde.

Die Konstruktion dieses Apparates ist aus der beigegebenen Figur leicht ersichtlich. Er besteht aus einem $2^{1 / 2}$ Liter fassenden zylindrischen Gefäss $A$, welches im Boden, nahe an der Wand, eine Öffnung besitzt. In dieser Öffnung ist das eine Ende eines durchbohrten Korkpfropfens, welches anderseits den erweiterten Hals des Hebers $C$ schliesst, befestigt; durch die Bohrung des Stopfens ragt das nach dem Niveau der Flüssigkeit verstellbare Abflussrohr $B$ aus dem Heber in das Gefäss $A$ hinein. Der Heber $C$ ist unten kugelförmig erweitert und mit einem Ablasshahn versehen. Sein absteigender Schenkel $G$ mündet tief in den Siedekolben $D$ ein. Durch die zweite Bohrung des diesen Kolben schliessenden Pfropfens steigt ein weites Rohr $E$ bis in den Rückflusskühler $F$ auf, dessen unteres Ende durch einen doppelt durchbohrten Stopfen geschlossen ist. Durch die eine Bohrung geht das Steigrohr $E$, durch die andere das für den Abfluss des kondensierten Äthers bestimmte engere Rohr H. Dieses Rohr $H$ erweitert sich nach unten in Form einer kleinen Glocke, welche in das ebenfalls glockenförmige Gefäss $I$ taucht. Letzteres Gefäss hat in der Mitte des Bodens eine Öffnung, in welcher das obere Ende des hohlen Rührers $K$ wasserdicht befestigt ist und ist, mit Wasser so hoch wie der Kühler ragt, angefüllt. Diese Einrichtung stellt einen luftdichten Wasserverschluss dar, dessen unterer Teil eine rotierende Bewegung um seine vertikale Achse, welche durch den Rührer geht, ausführen kann. Der Rührer ist in der hohlen Achse der auf dem Deckel $L$ montierten Transmissionsscheibe $S$ mit Hilfe einer Klemmschraube fixiert; sein unteres Ende hat die Gestalt eines $T$, dessen wagerechte Schenkel seitlich eine Reihe feiner Löcher tragen. Der Deckel $L$ ist aus Metall und ist mit einem doppelten Kragen, welcher die Gefässwand eng umschliesst, versehen. Ausser der Öffnung, welche als Lager für die Achse der Scheibe $S$ eingerichtet ist, besitzt er noch eine zweite zur Einfüllung der Flüssigkeiten in das Gefäss $A$. Während der Extraktion bleibt der Deckel, um Ätherverlust zu vermeiden gut verschlossen.

Um den Apparat nun in den Betrieb zu setzen, wird der angesäuerte Harn in das Extraktionsgefäss $A$ eingegossen und mit 
Äther etwa $2 \mathrm{~cm}$ hoch überschichtet. Das Abflussrohr $B$ wird durch Verschieben so gestellt, dass sein oberes Ende in einem Niveau mit der Ätheroberfläche steht. Dann wird noch so viel Äther zugefügt, dass er durch das Rohr $B$ in den Heber und von dort in den Siedekolben fliesst und letzteren so weit anfüllt, bis die Spitze des Heberschenkels $G$ eben bedeckt ist.

Der Apparat wird darauf geschlossen, der Äther in $D$ mit Hilfe eines Wasserbades zum Sieden gebracht und die Scheibe $S$ mit irgendeinem Motor in Verbindung gesetzt. Der Ätherdampf steigt durch $E$ in den Kühler; der kondensierte Äther fliesst durch $H$ in den Verschluss $I$ und von dort in den hohlen rotierenden Rührer, um durch die Bohrungen des letzteren in den Harn zu gelangen. Dort steigt er bis zur Oberfläche und fliesst durch das Abflussrohr $B$ in den Heber $C$ und endlich in den Siedekolben zurück. Wird etwas Harn mitgerissen, was selten vorkommt, so setzt er sich in der kugeligen Erweiterung des Hebers ab und kann durch den Hahn abgelassen werden. Auf diese Weise wird der Übergang von Harn in den Siedekolben vermieden.

Um das Ende der Extraktion zu erkennen, entnimmt man durch den Ablasshahn $15-20 \mathrm{ccm}$ Äther und prüft ihn nach Verdunstung bei gewöhnlicher Temperatur auf Salizylsäure. Ist die Reaktion negativ, so kann man sicher sein, dass der Harn nichts mebr enthält.

Der Apparat arbeitet sehr prompt und ohne nennenswerten Ätherverlust. Der gute Nutzeffelit wird durch die Rotation des hohlen Rührers bedingt; in der Tat, die feinen Äthertröpfchen, welche aus dem Rührer kommen, steigen nicht direkt an die Harnoberfläche, sondern sie werden durch den Harn verteilt und herumgewirbelt, so dass sie Gelegenheit haben, sich mit den Bestandteilen, welche man isolieren will, zu sättigen.

Die Ergebnisse meiner Resorptionsversuche sind nun folgende:

1. Gaultheriäol. Harn des ersten Normaltages: Brombindungsvermögen in $1 / 10$ norm. Thiosulfatlösung $17,5 \mathrm{ccm}$. Harn des zweiten Normaltages: Brombindungsvermögen: $16,8 \mathrm{ccm}$. Am dritten Tage, morgens 6 Uhr Einreibung von $2 \mathrm{~g}$ Gaultheriaöl. Die Harne der dritten und vierten Tage werden vereinigt und weisen ein Brombindungsvermögen von $96,25 \mathrm{ccm}$ auf. Zieht man den mittleren Wert von 2 Normaltagen, also $17,5+16,8=34,3 \mathrm{ab}$, dann bleiben $61,95 \mathrm{ccm}$, welche auf ausgeschiedene Salizyl- bzw. Salizylur- 
säure zurückzuführen sind. Da jeder Kubikzentimeter 1/10 norm. Thiosulfatlösung, wie wir eben gesehen haben, 0,00276 Salizylsäure entspricht, ist hiër $0,00276 \times 61,95=0,1709 \mathrm{~g}$ ausgeschieden worden. Das Molekülargewicht von der Salizylsäure ist 138, dasjenige des Gaultheriaöls 152. Die eliminierte Salizylsäuremenge stammt demnach von $0,1883 \mathrm{~g}$ Gaultheriaöl, welches durch die Haut resorbiert worden ist. Da $2 \mathrm{~g}$ eingerieben wurden, beträgt die perkutane Resorption des Methylsalizylats ca. 9,4\%.

2. Amylsalizylat. Die Berechnung wurde auf dieselbe Weise ausgefürt; ich brauche demnach die Details der Analyse nicht wiederzugeben; auch möchte ich nochmals betonen, dass die gefundenen Werte keinen Anspruch auf absolute Genauigkeit haben.

Von $2 \mathrm{~g}$ eingeriebenen Amylsalizylat wurden etwas mehr als 0,05 g resorbiert, das sind $\mathbf{2 , 6} \%$.

3. Mesotan, unzersetzt. Von $2 \mathrm{~g}$ wurden in einem Versuch $0,162 \mathrm{~g}=8,1 \%$, in einem anderen $0,153 \mathrm{~g}$, also $7,6 \%$, resorbiert.

4. Mesotan, zersetzt. Von $2 \mathrm{~g}$ wurden $0,48 \mathrm{~g}$ resorbiert, das sind $24 \%$.

5. Glykolmonosalizylat. Von $2 \mathrm{~g}$ wurden $0,318 \mathrm{~g}$, also $15,9 \%$, resorbiert.

6. Glykolmonosalizylat, mit gleichen Teilen Spiritusvorlauf verdünnt. Von $2 \mathrm{~g}$ Ester, welche eingerieben wurden, wurden $0,407 \mathrm{~g}$, das sind $20^{\circ}, 0$, resorbiert.

Fassen wir diese Ergebnisse zusammen, so sehen wir das die perkutane Resorption:

$$
\begin{aligned}
& \text { des Amylsalizylats . . . . . . ca. 2,60, } \\
& \text { des Methyksalizylats. . . . . . ca. } 9,4 \% \text {, } \\
& \text { des unzersetzten Mesotans . . . . ca. } 8 \% \text {, } \\
& \text { des zersetzten Mesotans . . . . ca. } 24 \% \text {, } \\
& \text { des Glykolmonosalizylats . . . . . ca. } 15,9 \% \text {, } \\
& \text { des verdünten Glykolmonosalizylats . ea. } 20 \% \text { beträgt. }
\end{aligned}
$$

Es sind, wie man sieht, grosse Unterschiede in der Aufnahmefähigkeit der untersuchten Ester durch die unversehrte Haut vorhanden; sie finden eine plausible Erklärung in den physikalischchemischen und physiologischen Eigenschaften dieser Substanzen, wie ich es jetzt zeigen will. 
1. Am spärlichsten resorbiert wurde das Amylsalizylat; dieser Ester zeichnet sich durch eine sehr schlechte Wasserlöslichkeit $(0,0039 \%)$, einen ungünstigen Teilungskoeffizienten von öl zu Wasser (25600), eine langsame Alkalispaltbarkeit $(0,27 \%$ in der ersten Stunde) aus. Die Flüchtigkeit ist zu gering, um irgendeine Rolle zu spielen; die innere Reibung ist stärker als beim Gaultheriaöl und die Oberflächenspannung kleiner.

2. Das Methylsalizylat und das unzersetzte Mesotan werden fast in den gleichen Verhältnissen resorbiert $(8-9 \%)$. Beide sind löslicher in Wasser als das Ulmaren; diese Löslichkeit ist für beide wenig verschieden $(0,072$ und $0,091 \%)$ und ebenso ihr Teilungskoeffizient (1380 und 1095); die Alkalispaltharkeit ist auch eine viel bessere $(2,2$ und $4,7 \%$ in der ersten Stunde). Durch diese Eigenschaften wird die ausgiebigere Resorption gegenüber dem Amylsalizylat bedingt. Die leichtere Verseifung des Mesotans, seine Zersetztlichkeit schon durch Wasser allein hätte aber eine stärkere Resorption dieses Esters im Vergleich mit Gaultheriaöl, erwarten lassen. Die geringe innere Reibung, die stärkere Oberflächenspannung des Methylsalizylats erlauben ihm ein rasches Imbibieren der Haut und ein schnelleres Eindringen in ihre Poren; das Mesotan dagegen hat von den vier Estern die geringste Oberflächenspannung; es ist auch sozusagen gar nicht flüchtig, während das Gaultheriaöl, wie wir gezeigt haben, eine wenn auch schwache, so doch zu beachtende Flüchtigkeit besitzt. Diese sekundären Faktoren gleichen beim Methylsalizylat den Vorsprung, den das Mesotan durch seine bessere Spaltbarkeit hat, zum Teil aus. Ausserdem muss man noch in Erwägung ziehen, dass das Mesotan in Berührung mit dem Schweiss, dank seiner Zersetzlichkeit durch Wasser, schon in seine Komponenten teilweise zerfällt, ehe es die äussere Hautschicht passiert bat. Nun wird die dabei entstehende Salizylsäure, wie bekannt, von der Haut sehr spärlich aufgenommen, weil sie in der Schweissflüssigkeit sich in Natriumsalizylat umwandelt, welches in Öl bzw. Hauttalg fast. unlöslich ist. So wirkt die Zersetzlichkeit des Mesotans seiner Resorption in gewissem Grade entgegen.

3. Das Glykolmonosalizylat wird am besten resorbiert $(16 \%)$, wenn wir von dem zersetzten Mesotan absehen. Das steht vollkommen im Einklang mit seiner guten Wasserlöslichkeit $(0,968 \%)$, seiner relativ schwächeren Öllöslichkeit $(16 \%)$, seinem infolgedessen 
sehr günstigen T'eilungskoeffizienten $(16,5)$ und endlich mit seiner Alkalispaltbarkeit, welche in der ersten Stunde dieselbe wie diejenige des Mesotans ist $(4,7 \%)$.

Ein seine Resorption erschwerendes Moment hat das Glykolmonosalizylat in seiner Dickflüssigkeit; dies lässt sich schon aus der Tatsache erkennen, dass die Eisenchloridreaktion im Harn in der ersten Stunde nach der Einreibung etwas stärker ausfällt mit Gaultheriaöl und Mesotan als mit Glykolmonosalizylat. Die Verdünnung letzteren Produktes mit dem dünnflüssigen Vorlauf erhöht seine Resorption von 16 auf $20 \%$. Da diese Mischung mit Vorlauf keine Reizwirkung und keine nennenswerte Hyperämie an der Applikationsstelle hervorruft, ist die bessere Resorption aller Wahrscheinlichkeit nach nicht allein auf eine regere Zirkulation in den Tegumenten zurückzuführen, sondern auch auf eine schnellere und ausgiebigere, durch die Verminderung der Viskosität bedingte Benetzung der Hautoberfläche. Die durch die starke innere Reibung entstandene Resorptionsverzögerung macht sich nur in der ersten Zeit nach der Einreibung bemerkbar; später, wenn die Haut benetzt ist, verliert dieser Faktor an Bedeutung, was schon daraus hervorgeht, dass das Glykolmonosalizylat in grösserer Menge als die beiden anderen Ester aufgenommen wird.

4. Fin wichtiger Faktor für die perkutane Resorption ist ohne Zweifel die Reizwirkung. Ein zersetztes Mesotan, welches die Haut reizt, starkes Jucken nnd Hyperämie hervorruft, wird zu 24\% resorbiert, $3 \mathrm{mal}$ so reichlich als das frische nicht zersetzte Mesotan. Diese rasche und stärkere Aufnahme lässt sich gut nachweisen, wenn man die resorbierten Mengen in den 5 ersten. Stunden nach der Einreibung bestimmt. Dieselben betragen für das zersetzte Mesotan ca. 7,4\%, während sie für das Glykolmonosalizylat nur etwa 4,7\% erreichen.

Die Hautreizung, welche die Resorption so intensiv befördert, wird aber nicht immer erwünscht; bei empfindlichen Tegumenten ist sie sogar kontraindiziert. Sie darf demnach nur als gelegentlicher Hilfsfaktor für die perkutane Resorption gelten.

Fragen wir uns jetzt, welcher von den untersuchten vier Salizylsäureestern sich am besten fürdie externe Therapie eignet, so erscheint mir das Glykolmonosalizylat als derjenige, welcher die meisten Vorzüge bietet. Ohne auf die Haut Reizerscheinungen her- 
18 E. Impens: Über die perkutane Resorption einiger Ester der Salizylsäure.

vorzurufen, wird er am reichlichsten resorbiert. Ausserdem ist er vollkommen geruchlos, während Ulmaren und besonders Gaultheriäl durch die Dämpfe, welche sie verbreiten, den Patienten und seine Umgebung stark belästigen.

Diese Gründe haben mich veranlasst, das Glykolmonosalizylat, welches von den Farbenfabriken vorm. Friedr. Bayer \& Co. dargestellt und unter dem Namen Spirosal in den Handel gebracht wird, für die klinische Prüfung zu empfehlen. 